\title{
A CELL SPACE EMBEDDING OF SIMULATED LIVING CELLS*
}

\author{
E. D. Goodman, R. Weinberg and R. A. Laing \\ Department of Computer and Communication Sciences, University of Michigan (USA)
}

(Received: 10 June, 1970)

SUMMARY

ONECELL, a computer simulation of the metabolism of a single cell, has been embedded in CELLSPACE, a tessellation model for expressing and manipulating intercell relationships. ONECELL and CELLSPACE parameters can be set so as to achieve normal slow monolayer growth of cells, or abnormal rapid multilayer growth. The biochemical basis of such phenomena is explicit, and can be varied at will.

\section{SOMMAIRE}

ONECELL simulation par ordinateur du métabolisme d'une cellule unique a été implanté dans 'CELLSPACE' modèle disposé en damier pour exprimer et pour manipuler les connexités intercellulaires. On peut reglér les paramètres de 'ONECELL' et de 'CEILLSPACE' de façon à obtenir la lente croissance normale et à couche unique de cellules, ou la croissance anormale à plusieurs couches. La base chimique de tels phénomènes est clair et on peut la varier à volonté.

\section{INTRODUCTION}

In 1968, Weinberg (1968) developed ONECELL, a system for computer simulation of the biochemistry of a single living cell. In order to investigate interactions among

\footnotetext{
* This research was supported by the National Institutes of Health and the National Science Foundation.

Papcr presented at the 1970 Summer Computer Simulation Conference, Denver, Colorado (USA) sponsored by ACM/SHARE/SCi.
}

Biu-Mfelical Computing (2) (1971)-C Elsevier Publishing Company Itd, England-Printed in Great Britain 
developing populations of cells, we have embedded ONECELL in a tessellation space (an automata-theoretic cell space with (in our case) a fixed neighborhood relationship). We simulate the tessellation space using a flexible set of computer assembly-language routines; this set of routines is called the Cellular Space Simulator (CELLSPACE). The combined system (ONECELL + CELLSPACE) is being used to study the biochemical events responsible for normal and abnormal development in groups of living cells.

Information in the ONECELL + CELLSPACE system is handled as follows:

(1) In order to determine its metabolic status, each ONECELL must sense and react to the environment (nutrients, etc.) at its CELLSPACE location. (The individual cell's metabolic behavior has been adjusted to agree with laboratory data.)

(2) Each ONECELL must communicate information of its internal metabolic state to its self-replication mechanism. (The experimental evidence of the Helmstetter-Cooper model of replication has been incorporated.)

(3) CELLSPACE must calculate the spatial effects of cell growth (both in terms of cell size and numbers of cells) and communicate the new spatial (as well as nutritional) properties to each ONECELL location. (The tessellation automaton concept of von Neumann as implemented in a computer by R. Brender (1970) and T. Schunior has been employed here.)

By suitably choosing the CELLSPACE condition for inhibition among neighboring cells, it was possible to achieve slow, monolayer growth by 'normal' cells, while 'abnormal' cells grew more rapidly and in multiple layers.

Relatively minor changes in the metabolic pathways can produce markedly different growth patterns in colonies of living cells. The biochemical basis of such global effects in cell growth can be examined in detail, since the underlying biochemical equations responsible for these effects are completely explicit and can be varied at will.

MODELING THE PROCESSES OF A SINGLE CELL

\section{Background}

The advances in molecular biology in the past fifteen years have greatly increased our understanding of the basic subsystems of the living cell. DNA has been found to be the molecule on which the cell records information to be passed on to the next generation. M. H. F. Wilkins, James D. Watson and Francis H. C. Crick have established the precise structure of the DNA molecule. The molecular code used by DNA to record the information to be passed on has been successfully analysed. Viable hypotheses have been formulated for the translation of genetic information into cellular activity and structure. Experiments have yielded support for specific theories concerning the control of cellular activity at the level of DNA function 
(for example Davis et al., 1968; Weinberg, 1956, 1960, a, b; Weinberg and Boyer, 1965). Control mechanisms have also been discovered which govern a given cellular process by modifications of molecules far removed from DNA. The interactions of the various subsystems analysed by the molecular biologists are becoming objects of study. These subsystems interact among themselves, and with the environment external to the cell.

One can study these complex interacting systems by formulating hypotheses in the form of logical and analytic equations, and embodying these in a computer program. Environmental conditions (such as nutrient levels, temperature etc.) can be given to the computer as input data. A computer-program representation of the metabolic processes of a living cell yields as output a description of cell behavior (e.g. rates of metabolism, replication and cell division).

The availability of excellent and detailed experimental and theoretical analyses of the metabolic and informational systems operative in living and reproducing organisms, as well as newly developed powerful and convenient computer and system theory techniques today make possible excellent computer simulations of individual living cells (Weinberg and Zeigler, 1970).

\section{Simplifying Assumptions For ONECELL}

In our attempt to model a cell at the biochemical level we had to confront the complexity of the metabolic pathways present in even the simplest of cells. More than 3000 different kinds of molecules are present in a complex spatial and functional relationship. This complexity had to be drastically reduced to permit a simulation by a digital computer. To construct our simulation, we lumped molecules into pools, and considered (1) metabolic topology, (2) functional relationships among cell structures and chemicals, and (3) the type of experimental data available in the literature (Weinberg and Zeigler, 1970). (This process of lumping is further described and justified from a systems viewpoint elsewhere (Zeigler and Weinberg, 1970).)

Metabolic topology was considered, and an attempt was made to lump together as a single entity only those molecules which could be drawn adjacent to one another on the metabolic map (Figs. 1,2). For example, in a pathway $\mathrm{A} \rightarrow \mathrm{B} \rightarrow \mathrm{C}$, chemicals $\mathrm{A}, \mathrm{B}, \mathrm{C}$, might be partitioned into $\mathrm{A}$, and $\mathrm{BC}$, but would not be lumped into $\mathrm{AC}$ and $B$.

Functional relationships between groups of molecules were extremely important, and the molecules lumped together in any one model entity were, in some way, functionally a unit. Thus, all the molecules produced in the breakdown of sugar to $\mathrm{CO}_{2}$ and water to produce energy were lumped in this model since they could be considered functionally as molecules intermediate in a chemical pathway used to produce energy. Later models will employ more refined partitions, for example to capture the subtle and important relationships between molecules at different points in the glycolytic pathway, citric-acid cycle and cytochrome system. 


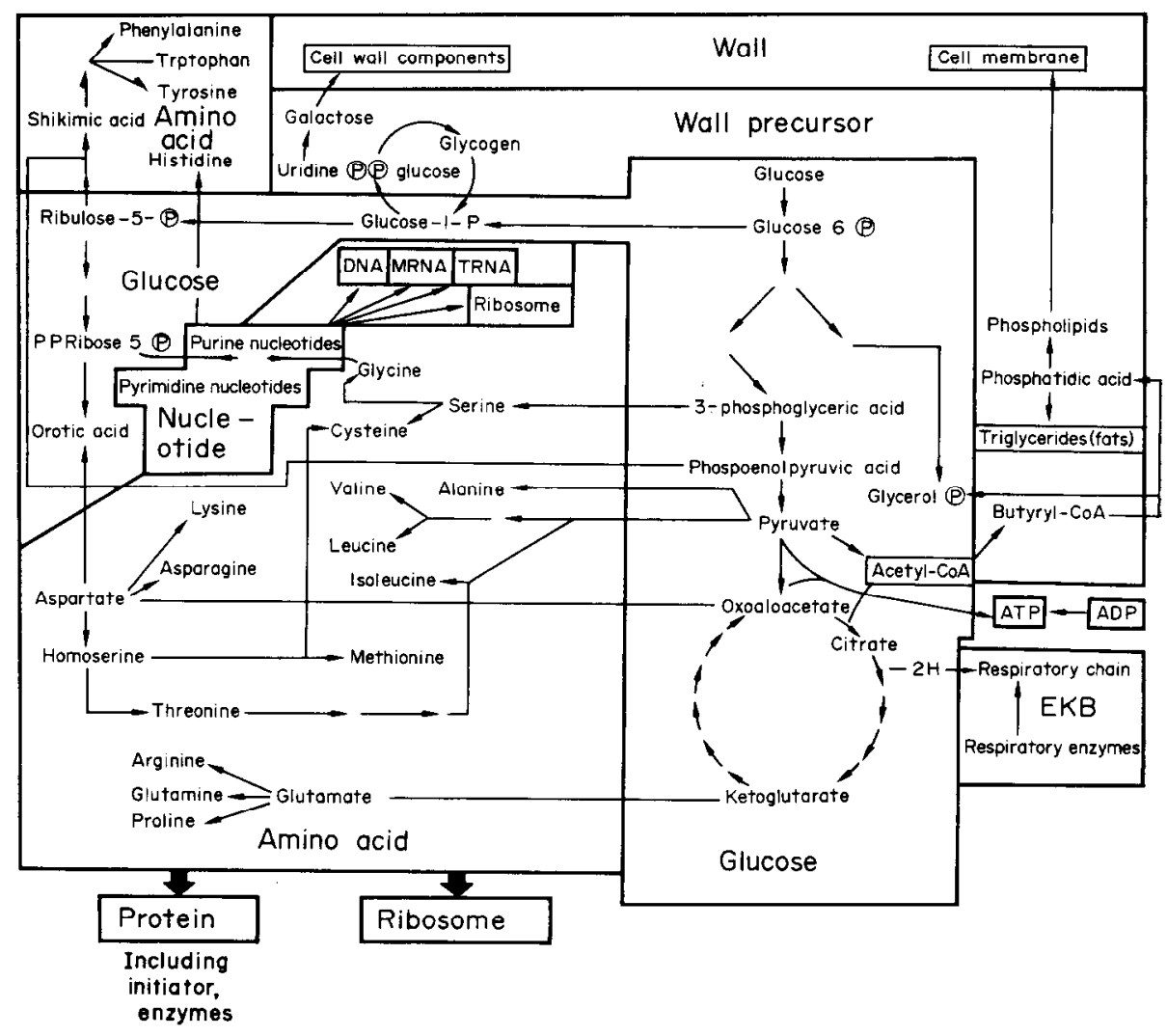

Fig. 1. Some of the primary molecular constituents and metabolic pathways in the E. coli cell are shown. These constituents are shown grouped together into 'pools', e.g. GLUCOSE, AMINO ACIDS, etc., which serve as the entities for a model of the cell.

Experimental data were often available for large chemical pools, e.g. products of glycolysis and the citric-acid cycle, making these separate entities logical candidates for grouping into single entities in this model of the cell.

Because the most complete data were available for Escherichia coli, the parameters were set using $E$. coli data. While it would have been possible to change many parameters to more closely fit data for higher (e.g., mammalian) cells, it was thought to be more important to use a single consistent set, and $E$. coli seemed to be a good choice.

We are using the model to test hypotheses concerning intercell control mechanisms. Many of these mechanisms are not characteristic of $E$. coli, but the chemical pathways and processes of $E$. coli have an underlying similarity to those of a broad class of cells, particularly at the level at which our model operates. It is therefore 


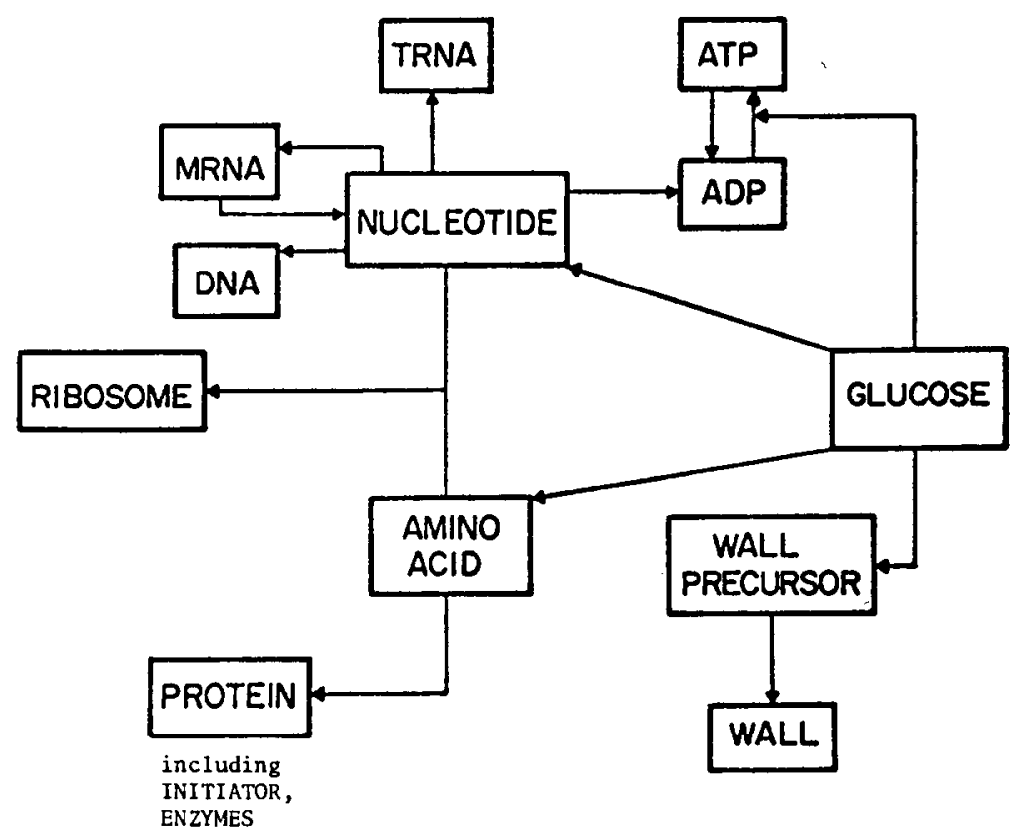

Fig. 2. The primary chemical pools used in the model are shown. The arrows indicate the direction of the flow of material between pools. The topology of this flow preserves the metabolic topology of Fig. 1, in a manner explained in the text.

felt that insight gained using these $E$. coli parameters will be of some general applicability in studying colonies composed of other cell types.

\section{The Resulting Model: ONECELL}

ONECELL can be described by its state together with its transition function. The state of the ONECELL is described (1) by the concentrations of 30 internal chemical pools, (2) by the genetic apparatus, and (3) by the cell volume (Fig. 3). The transition function (Fig. 4) used to obtain the state of the cell at the next time

\begin{tabular}{c} 
Concentrations of Chemical Pools, PRDC(1), ., PRDC(10) \\
\hline Concentrations of Enzymes, EK(1), ., EK(10) \\
\hline Concentrations of Messenger RNA's, RNK(1), ., RNK(10) \\
State of DNA Replication Sites, Amount of DNA, \\
Amount of Initiator IN \\
Total Volume of Cell \\
\hline Number of Cells Represented by this ONECELL
\end{tabular}

Fig. 3. State variables in ONECELL. 
TRANSITION FUNCTION

(for calculating the state of the system in the next time step from the present state)

A. The differential and Boolean equations relate concentrations of variables at a given time to the concentrations of those variables DT seconds later.

An example:

1. For AA, the amino acid pool, enzyme $E K(2)$ is needed to catalyse the production of AA from glucose, and ATP is used as an energy source. At the same time, AA is lost as it is used for the production of RIB and PRTN. This is expressed in the equation: $\mathrm{DAA}=\mathrm{K}(2) \star \mathrm{GLUC} \star \mathrm{DT} \star \mathrm{EK}(2) \star \mathrm{ATP}-1 . \mathrm{E} 6 / 102, \star \mathrm{DRIB}-(4 . \mathrm{E} 4 / 102) \star \mathrm{DPRTN}$ production of AA from loss of AA to loss of AA to PRTN GLUC RIB

2. RNK(2) produced EK(2) from AA under the direction of DNA, using ATP for energy: $\operatorname{DEK}(2)=\mathrm{K}(7) \star \mathrm{AA} \star(\mathrm{RNK}(2) / \mathrm{MRNAO}) \star \mathrm{DT} \star \mathrm{EK}(7) \star \mathrm{ATP}$

3. RNK(2) itself was produced from NUC under the direction of DNA, catalysed by EK(8), using ATP for energy. RNK(2) decayed spontaneously at the same time, producing some loss of RNK(2) already present:

$\operatorname{DRNK}(2)=(\mathrm{KB}(2) \star N U C \star \mathrm{DNA} \star \mathrm{EK}(8) \star A T P-K D R N K \star \mathrm{RNK}(2)) \star \mathrm{DT}$ production of RNK(2) decay of RNK(2)

B. Allosteric modification of enzymes is simulated by modifying the rate constant which characterises all different forms of any enzyme associated with a particular reaction.

C. Repression of messenger RNA directing the production of a particular enzyme occurs.

D. Response of the replicon to the state of the cell is calculated.

E. Permeability to nutrients and to excitatory or inhibitory substances in the surrounding cells or the growth medium is simulated.

Fig. 4. Elements of the transition function.

step of the simulation from a given time step consists of difference equations and Boolean expressions describing (1) enzyme catalysed chemical reactions, (2) allosteric modification of enzymes, (3) repression of messenger RNA-production, (4) self-replication of DNA under genetic controls, and (5) permeability of the cell to the chemical pools represented in the simulation. A detailed description of an earlier version of ONECELL is available (Weinberg and Berkus, 1969).

Input to ONECELL consists of the concentrations of chemicals in various nutritional environments in which ONECELL is assumed to be growing. Output from ONECELL consists of state descriptions during successive time increments.

\section{The Model of DNA Replication}

Self reproduction in ONECELL is controlled by an initiator substance in association with the cell's genetic complex; the Helmstetter-Cooper model for control of DNA replication is incorporated as part of our genetic apparatus. The HelmstetterCooper theory provides the most sophisticated model of DNA replication presently available (Helmstetter et al., 1968).

Basically, the Hclmstetter-Cooper model asserts that the life sequence of a cell begins with the accumulation of a protein substance which is the 'initiator' of chromosome replication. When a threshold amount of initiator substance has accumulated, replication of DNA commences at a fixed end-point on the chromosome, and the replicating process proceeds to traverse the entire length of the chromosome. At the completion of DNA replication, a sequence of further cell 
events follows, terminating in physical cell division. Even before completion of cell division, however, a new DNA replication cycle may have begun-this will depend on the amount of accumulation of initiator substance by that time. It is thus possible for the DNA replication process to be taking place simultaneously at several points on the chromosome, as represented by the multiple forks shown in Fig. 5.

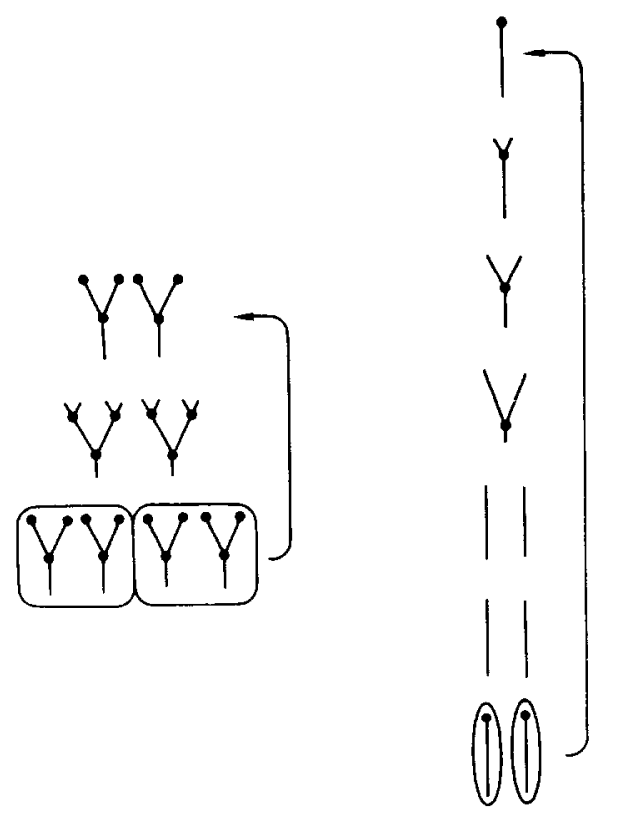

Fig. 5. DNA replication model for 20- and 60-minute cells (after Helmstetter et al., 1968).

The ultimate rate of cell division is thus a function of the periodic attainment of a sufficient fixed amount of the initiator substance which is synthesised continuously and which can be affected by the richness of the cell's nutritional environment.

Under optimal $\mathrm{pH}$, temperature, and cell density conditions, 'log phase' $E$. coli cells double one to three times per hour. The rate is a function of the medium in which the cells are grown. Although cell life outside this 'log phase' range should not be ignored, much of what we wish to know of cell life takes place under these conditions. In particular, many anomalies of control of cell behavior (including some 'cancerous' behavior) take place while cells have a 'normal range' nutritional environment.

Implementing the Replication Model in ONECELL

ONECELL's rate of initiator production is a function of the metabolic rates along the various simulated internal biochemical pathways; these rates in turn are dependent on the nutritional levels supplied to ONECELL. 
In ONECELL we can simulate the effect of growth in three different media: (1) mineral glucose medium, (2) a medium to which amino acids (casamino acids) have been added (thus obviating the necessity for the cell to synthesise these itself), and (3) a still richer medium which contains both amino acids and nucleosides (broth). In ONECELL these three nutritional environments result in steady-state cell division rates of once every fifty minutes, once every twenty-eight minutes, and once every twenty-five minutes respectively. In effect, through the mechanisms of the replicon, we simulate the production of varying amounts of DNA, depending on the richness of the nutritional environment, and the DNA amount in turn has both internal biochemical consequences and consequences for the overall celldivision rate.

The replication mechanism in ONECELL may be summarised as follows: the rate of initiator production depends on concentration of internal pools of amino acid, ATP, and the enzyme catalysing the production of initiator from these substrates. This internal information from ONECELL's cytoplasm simulation is transmitted to the replication apparatus. When sufficient initiator substance is produced, replication begins at a DNA initiation site. Replication of the DNA molecule takes 40 minutes to complete. After the replication of a DNA molecule is completed, the cell will proceed to divide; the physical division of a cell into two cells occurs after 20 minutes, and each of the two new cells receives half of the DNA present in the original simulated cell.

\section{DESCRIPTION OF CELLSPACE}

\section{Background}

In order to present a mathematically rigorous proof of machine self-reproduction, John von Neumann (1966) introduced the idea of cellular automaton systems. His cellular model can be described in an informal way as an infinite chess board, each square of which contains a copy of the same (in his case, 29 state) finite automaton. One decides on some initial setting of state for each of the automata occupying the squares. This determines the state of the whole system at the initial moment of time. At subsequent discrete moments of time, the state of the automaton in each square at time $t+1$ is uniquely determined by its state at time $t$ and the states, also, at time $t$, of its four 'North, East, South, West' neighbors. Usually all but a finite number of the automata in the squares are initially set at a quiescent state. This region of quiescent states is usually interpreted as the unorganised external environment into which the developing cellular organism (expressed in the active automaton squares) may penetrate.

Using this system, von Neumann was able to demonstrate constructional universality (including self-reproduction) for non-biological systems. Cellular automaton systems can, however, be used to express the behaviors of many other systems 
(including the biological) in which 'modest' initial conditions and local neighborhood relations may grow to have global effects.

One need not be limited to 'checkerboard' two-dimensional cellular spaces. The finite automata in each cell space need not be limited to the 29 state devices von Neumann found sufficient. The neighborhood relationship may involve other than 'cardinal direction' cells. Although our application does use the 'cardinal direction' neighborhood, the number of states in each of our finite automata is very large, each state representing a possible biochemical state of a living cell.

At the Logic of Computers Group at the University of Michigan, we have engaged in a number of theoretical analyses of cellular spaces and other parallel computation spaces (Brender, 1970; Burks, in press; Codd, 1968; Zeigler, 1970). We have also designed and implemented a cell space computer simulation system (CELLSPACE) in which cell array size, dimensionality, transition function, and neighborhood relations can be easily assigned and easily modified (Brender, 1970).

\section{Modeling a Colony}

The concept of a cell space captures in the abstract many fundamental properties of populations of living cells. Almost all of the cells in an individual organism have the same genetic complement; all of the coordinates of the cell space have the same transition function.

The complex biochemical interactions leading to the complete adult organism are functions of the chromosome complement of the original zygote, together with the neighborhood relationships established as the cell reproduces. Self-reproduction and specification of neighborhoods are natural concepts in cell spaces, and make cell spaces good vehicles for studies of the development of a few living cells into large numbers of interacting cells.

In order to maintain the potential for comparing the output of the simulation with laboratory data, the simulation was designed to model the growth of cells on a solid medium (in a Petri dish, for example). Because of the obvious need to model large colonies using as few simulated cells as possible in representing them, it was decided that a simplifying assumption of cylindrical symmetry about the center of the colony should be made. Under this assumption, it is possible to represent the three-dimensional colony in a two-dimensional cell space. Figure 6(a) shows the pattern of 31 cells which has been used for the initial experiments. Each point in the figure represents a possible position of a cell in a vertical section through a radius of a colony; i.e. if the figure were rotated around the left-hand vertical row of points, the shape of the colony represented would be traced out. Of course, the arrangement of these 31 points is such that it can accommodate a variety of colony shapes; however, the right-hand 'diagonal' edge is a boundary since colonies would not grow with a vertical edge. In practice, the simulation must be stopped (or the space enlarged) when cells reach this boundary. The bottom row represents the layer of cells resting directly on the growth medium. When the 
(a)

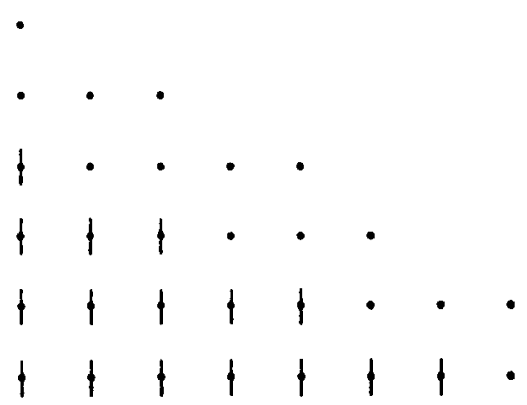

(b)

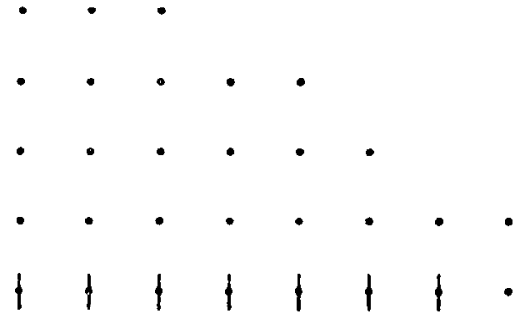

(d)

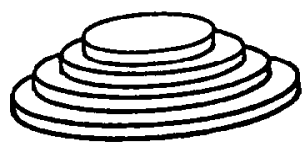

(c)

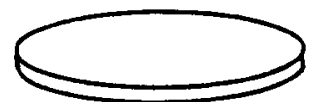

(e)

Fig. 6. Preliminary experiments using ONECELL + CELLSPACE-(a) the space; (b) fast, multilayer growth; (c) colony shape represented in (b); (d) slow, monolayer growth; (e) colony shape represented in $(\mathrm{d})$. 
simulation is started, only the lower-left-hand point actually represents cells, the other points will come to represent cells when adjacent cells divide and daughter cells assume positions at the new points. It should be made clear that the few points in the space represent large numbers of cells in the colony; in fact, each represents all of the cells in the colony at a particular distance from the center and height from the dish. Thus these 31 cells (which can readily be expanded up to 50 or more) are used to simulate colonies of several hundred cells, and can simulate more cells if less detailed representation is acceptable. The position of a cell in the cellular space (e.g., bottom row, third from left) is not necessarily indicative of absolute position of the modeled cells in the colony; it indicates relative position. However, a better idea of the geometry or shape of the colony is obtained by computing, for each cell in the space, the distance of the cells it represents from the center of the colony. This is done using the information stored at each point concerning the number and volume of the cells it represents.

\section{Interactions Among Cells}

Interactions among cells in the colony are modeled by allowing each cell in the space to sense portions of the states of the cells which surround it, or are in its 'neighborhood'. The neighborhood is here defined to be the four nearest points; i.e. above, below, left (toward the center of the colony), and right (toward the outer edge). Of course, each cell also has access to the state of the other cells in its own radius, since they are represented by that cell itself.

It is important to notice some properties of information transfer in a cell space. With the neighborhood relationship defined as above, information can 'flow' at a rate of only one cell per time step. That is, a change of state in cell A two points away from cell B cannot influence cell B immediately, but must spend one time step in influencing an intervening cell. In a sense, one might expect that this consideration would prove to be very restrictive; its effect seems to be minimal. It does not prevent one from modeling such 'global' types of information transfer as nutrient diffusion; all it requires is that the time step be sufficiently small that the effect of the diffusion is not significantly altered by the time required for the effect of a change to propagate throughout the space.

\section{COMPUTER IMPLEMENTATION OF ONECELL AND CELLSPACE}

The simulation computation has been carried out on an IBM 1800 equipped with $16 \mathrm{~K}$ of core storage and associated disk memory. CRT pictures of the states of the simulation are made available to the investigator on a DEC display driven by a PDP-7. The PDP-7 is, in turn, connected to the IBM 1800 by means of a specially designed high-speed interface. Investigator interaction with the simulation during 
run-time is carried out through the teletype keyboard of the PDP-7 and the display's light pen.

The cell-space simulator CELLSPACE allows the user to specify a finite cellular space by supplying its transition function, neighborhood relationships, initial state, and other such data. For our application, CELLSPACE is supplied a call to ONECELL's Fortran routines which furnish a part of CELLSPACE's transition function. CELLSPACE also requires specification of certain parameters and procedures concerned with the implementation of itself - for example, translation tables for the visual display routines, an external cell procedure etc.

ONECELL is actually a general name for a set of programs with various capabilities. It consists of (1) a set of routines which are used to establish the parameters for a single cell by 'growing' the cell in each of three environments, (2) a set of routines for examining the behavior of a single cell in a single environment, of which a subset is used in the ONECELL + CELLSPACE runs, and (3) a set of routines for taking a 'snapshot' of the 1800 words which comprise the state of the cell and all of the associated parameters at any time in any environment, and storing this information on the disk for use in the ONECELL + CELLSPACE configuration. All of these routines are written in IBM 1800 Basic Fortran IV.

Typical operation of the combined ONECELL + CELLSPACE system begins with the establishment of the parameters of the cell which is to be the first cell in the colony. The routines to accomplish this (Link 1) are much too large for the $16 \mathrm{~K}$ core of our IBM 1800, so the program is written to allow five sections of Link 1 to be loaded on call, overlaying one another. These routines allow the user to obtain a variety of printed outputs or CRT display (controllable at run-time) as the cell is shifted through a variety of environments. When the desired environment for simulation in the cell space is reached, a data switch can be used to cause the entire COMMON area (containing both systems parameters and the current state of the cell) to be written to the disk. One can then choose (1) Link 2, to examine in detail the behavior of the cell in isolation (to check out new intracellular mechanisms etc.), or (2) Link 3, the first link of the ONECELL + CELLSPACE programs, to start simulating the growth of a clone of cells from the data for the single cell stored on the disk. Link 3 does certain data manipulation and debugging output, and calls Link 4 to perform the growth simulation in CELLSPACE.

Link 4 contains an IBM 1800 assembly-language program which specifies certain parameters and procedures for CELLSPACE and provides calls to the portion of ONECELL which performs the 'biochemical' transitions for each cell at each time step. Link 4 also includes Fortran subprograms and funclions for allowing interactions of various chemical pools among neighboring cells and interactions of the cells with the nutritional medium and the surrounding air. All output from Link 4 is via CELLSPACE, and is in the form of decimal digits displayed on the DEC 338 display scope associated with the PDP-7 computer. The entire simulation process carried out in Link 4 is under the user's control at run time. Transitions, 
output, parameter changes and all other manipulations are controlled either from the 338 using a light pen or from the PDP-7's teletype. The commands presently implemented include commands to:

(1) perform one transition step for each cell,

(2) back-up one step,

(3) perform transitions until halted,

(4) display the 31 cells on the CRT using the current display map,

(5) change the display map,

(6) change the scale or position of the display,

(7) save the external state vectors (for access by neighboring cells) on the disk,

(8) restore the external state vectors of all cells from the disk,

(9) save the internal state vector (biochemistry) for a cell on the disk,

(10) manipulate the internal state vectors stored on the disk,

(11) change selected parameters in the transition function.

In a sense, each cell in the ONECELL + CELLSPACE simulation is a copy of ONECELL, together with certain modifications to allow interactions with neighbors. It is not necessary, of course, to duplicate the transition function of ONECELL for each individual cell in the simulation, but rather to store the state of each cell in the space. Then transitions can be carried out for each cell in sequence by applying the transition function to each state vector in sequence. Of course, the next state of any given cell depends not only on its present state, but also on the present state of its neighboring cells in the space. In this implementation, however, only a portion of the state vector of the neighboring cells is available to the transition function. This is nccessitatcd by corc-storage limitations, but it should be clear from the following that it represents no real restriction on the capabilities of the simulation. The entire state vector for each cell (i.e. ONECELL's state vector) contains approximately 60 real variables (120 1BM 1800 words). Because it is desirable to be able to simulate as many cells as possible, this 60 -variable state vector is partitioned into two parts, the external state vector and the internal state vector. Only the external state vector for each cell remains in core storage at all times for access by neighboring cells. The internal state vector for a cell is copied from disk into core only to perform the transition for that cell. The external state vector consists (at present) of 16 words, those being 6 integer variables and 5 real variables. The use of 3 integer variables and 3 real variables may be decided upon prior to a simulation run, and thus a wide range of interactions among cells can be obtained using these variables.

Any variable in the external state vector is available as output on the display scope. Thus if it is desired to monitor some particular concentration in each cell, a single Fortran statement can be used to put that concentration in the external state vector for the course of the experiment. In running the simulation, one can 
display at any time any of the 11 external state variables. While other forms of output are certainly possible, they have not been found necessary as yet, and have not been implemented.

Setting up a particular experiment involves two operations: (1) placing the appropriate variables in the external state vector (for interaction among cells and for output), and (2) altering those equations in the transition function which allow the interaction variables to influence the state transition. All of this can be done in Fortran and is very simple to accomplish.

\section{POTENTIAL APPLICATIONS}

The range of problems open for study using ONECELL + CELLSPACE is broad. It is important that the simulation provide data which can be compared with laboratory data in order to make testable the hypotheses being modeled. Thus we wish to simulate as many of the observable properties of a colony of cells as possible. Among the properties within the scope of the ONECELL + CELLSPACE simulation arc:

(1) average rates of cell division in various environments,

(2) differences in cell-division rates in various parts of the clone,

(3) cell volumes as a function of nutrients and position,

(4) radius and height (shape) of the colony,

(5) concentrations of various diffusible and non-diffusible substances in different parts of the colony.

If we desire to study the effects of changes in cell internal biochemical pathways, this can be largely accomplished employing ONECELL alone. The great potential importance of the combined ONECELL + CELLSPACE systems instead lies in the study of the consequences of different conditions for interaction between cells. These interactions may be of several sorts, including:

(1) reduction of access to metabolites because of intervening cells,

(2) waste accumulation due to surrounding cells,

(3) intracellular reactions specifically induced or altered by cell wall contact with, or crowding by, neighboring cells, and

(4) intercellular diffusion or transport of chemical messengers, inhibiting or otherwise influencing cellular metabolism.

Interactions of types (3) and (4) above are of great interest to biologists at the present time, particularly in the study of the regulatory mechanisms which are altered in cancerous growth. For example, there may be a hypothesis that a particular chemical messenger, produced under certain circumstances, alters a particular 
biochemical pathway in cells into which it diffuses. One can simulate the action of the messenger using ONECELL + CELLSPACE and observe the effects it has on the growth of a colony, either in terms of the concentrations of various chemical pools in each cell or in terms of gross characteristics of the colony. An important feature of this simulation system is the opportunity for the experimenter to make changes in the amount or even the type of effect caused by the variables under study while the simulation is in progress. Heuristic exploration may be carried out by altering parameters as the simulation progresses. By repeatedly making alterations and observing their effects, one may be able to find parameters which yield the desired behavior.

\section{PRELMMNARY RESUISIS}

We shall present one experiment to illustrate the sorts of experiments which can be done with the system. The starting cell was initialised (in Link 1) for environment 1 , called 'minimal medium'. An inhibition function was programmed to act as follows: the cells in the colony were exposed to varying concentrations of a substance, call it INHIB, which affects the rate of production of IN, a protein which causes initiation of DNA replication upon accumulation of a certain amount of IN. The concentration of INHIB in each cell was made to depend upon the degree to which the cell was surrounded by other cells. Thus, cells on the outer periphery of the colony had relatively low concentrations in INHIB, while those in the center had relatively high concentrations. The effect of this inhibition was to change the entire character of the colony: without the inhibition it was a rapidly growing multi-layer colony; with the inhibition it became a slowly growing monolayer colony (see Fig. 6 (b)-(e)). It became clear that this sort of change (from multilayer to monolayer) is a function of both the amount of inhibition induced by neighboring cells, and also the underlying rate of growth as determined by the nutrients available in the simulated growth medium.

It may seem at first surprising that the multi-layer 'cancerlike' growth is the easier of the two growth patterns to implement in the cellspace; that is, our chief difficulty is to simulate the mechanisms which result in the more restricted 'normal' growth. However, this situation has a parallel in biological research. Often, the more difficult problem in studying regulatory aberrations is to learn the exact nature of the normal controls; once this is known, it is much easier to study the control aberrations which give rise to pathological conditions.

It is expected that further experiments of this sort will render explicit many implications of the ONECELL model, the Helmstetter-Cooper replicon, and the sorts of inhibition which may be programmed into the CELLSPACE + ONECELL system. 


\section{REFERENCES}

Brender, R. F., Ph.D. thesis, University of Michigan (1970).

BURKs, A. W. (ed.), Essays on Cellular Automata, University of Illinois Press, Urbana, Illinois (in press).

Cond, E. F., Cellular Automata, Academic Press, New York (1968).

Davis, B. D., Dulbecco, R., Elsen, H. N., Ginsburg, H. S., and Wood, W. B., Microbiology, Harper and Row, New York (1968).

Helmstetter, C., CoOPER, S., PIERUCCI, O., and Revelas, E., Cold Spring Harbor Symposia on Quantitative Biology, 33 (1968) p. 809.

von NeumanN, JoHn, Theory of Self-Reproducing Automata, Burks, A. W. (ed.), University of Illinois Press, Urbana, Illinois (1966).

WEINBERG, ROGER, J. Bacteriol., 72 (1956) p. 570.

WEINBERG, ROGER, J. Bacteriol., 79 (1960a) p. 612.

WEINBERG, ROGER, J. Bacteriol., 79 (1960b) p. 558

Weinberg, Roger, Bacteriol. Proc., G114 (1968).

WeInBerg, Roger and Berkus, MiCHAel, Technical Report 01252-2-T, University of Michigan, Ann Aurbor (1969).

WEINBERG, ROGER and BOYER, H., Genetics, 51 (1965).

WeInberg, Roger and Zeigler, B. P., J. Amer. Soc. Cybernet. (to be published, 1970).

ZEIGLER, B. P., presented at the 6 th International Congress on Cybernetics, Namur, Belgium, 1970. ZeIGler, B. P. and WeINBERG, RoGer, J. Theoret. Biol. (to be published, 1970). 